Comment. Math. Helv. 74 (1999) 53-62 0010-2571/99/010053-10\$1.50+0.20/0
(C) 1999 Birkhäuser Verlag, Basel

Commentarii Mathematici Helvetici

\title{
On nonpositively curved Euclidean submanifolds: splitting results
}

Luis A. Florit ${ }^{1}$ and Fangyang Zheng ${ }^{2}$

Abstract. In this article, we prove that a $n$-dimensional, non-positively curved Euclidean submanifold with codimension $p$ and with minimal index of relative nullity $\nu=n-2 p$ is (in an open dense subset) locally the product of $p$ hypersurfaces.

Mathematics Subject Classification (1991). Primary 53B25; Secondary 53C40.

Keywords. Isometric immersion, non-positively curved, Euclidean submanifold.

Let $f: M^{n} \rightarrow \mathbb{Q}_{c}^{n+p}$ be an isometric immersion from a Riemannian manifold into a complete simply connected Riemannian manifold of constant sectional curvature $c$ (superscripts will always denote dimensions). Denote by $\nu$ the index of relative nullity of $f$,

$$
\nu(x)=\operatorname{dim}\left\{X \in T_{x} M: \alpha_{f}(X, Y)=0, \forall Y \in T_{x} M\right\},
$$

where $\alpha_{f}$ stands for the vector valued second fundamental form of $f$. It is well known that having $\nu>0$ imposes strong restrictions on the manifold $M^{n}$ and on its isometric immersion $f$. In [F1], the first author proved the inequality $\nu \geq n-2 p$ when the sectional curvature of $M^{n}$ satisfies $K_{M} \leq c$ and gave several applications of this result. First let us show that this estimate is sharp.

Example. For each $i=1, \ldots, p$, let $S_{i} \subseteq \mathbb{R}^{3}$ be a negatively curved surface. Then the product $M^{2 p}=S_{1} \times \cdots \times S_{p} \subseteq \mathbb{R}^{3 p}$ satisfies the equality $\nu=n-2 p=0$.

More generically, let $M_{i}^{n_{i}} \subseteq \mathbb{R}^{n_{i}+1}$ be nowhere flat nonpositively curved hypersurfaces, $i=1, \ldots, p$. The Gauss equation tells us that the relative nullity $\nu_{i}$ of $M_{i}^{n_{i}}$ is $\nu_{i}=n_{i}-2$. Then, the product manifold $M^{n}=M_{1}^{n_{1}} \times \cdots \times M_{p}^{n_{p}} \subseteq \mathbb{R}^{n+p}$ also have $\nu=n-2 p$.

\footnotetext{
${ }^{1}$ Research partially supported by CNPq Brazil.

${ }^{2}$ Research partially supported by a NSF grant and an Alfred P. Sloan Fellowship. This project is also sponsored by the National Security Agency under grant \# MDA904-98-1-0036.
} 
The first author proved in [F2] a general splitting theorem for Euclidean submanifolds $f$ of nonpositive sectional curvature, under the additional assumption that the normal bundle of $f$ is flat. The main purpose of this paper is to drop that assumption in the borderline case $\nu=n-2 p$ to prove that the above example is essentially the unique one with minimal relative nullity index.

Theorem 1. Let $f: M^{n} \rightarrow \mathbb{R}^{n+p}$ be an isometric immersion into Euclidean space of a Riemannian manifold with nonpositive sectional curvature. Assume that $\nu=n-2 p$ everywhere. Then there exists an open dense subset $\mathcal{U} \subset M^{n}$ such that $\left.f\right|_{\mathcal{U}}$ splits locally as a product of $p$ Euclidean hypersurfaces, that is, for any $x \in \mathcal{U}$, there exist a neighborhood $x \in \mathcal{V} \subseteq \mathcal{U}$ and $p$ nowhere flat Euclidean hypersurfaces $f_{i}: M_{i}^{n_{i}} \rightarrow \mathbb{R}^{n_{i}+1}$ of nonpositive sectional curvature, such that

$$
\mathcal{V}=M_{1} \times \cdots \times M_{p} \quad \text { and }\left.\quad f\right|_{\mathcal{V}}=f_{1} \times \cdots \times f_{p}
$$

split.

First of all, note that when $f$ is analytic, the splitting occurs on the entire $M$. In the general case, each $n_{i}$ is constant in a connected components of $\mathcal{U}$, in fact, the universal covering space of any component of of $\mathcal{U}$ is the product of $p$ Euclidean hypersurfaces. However, there are examples in which the $n_{i}$ 's are not constant in the entire $\mathcal{U}$. Secondly, it is interesting to observe that, from Theorem 1 of $[\mathrm{M}]$ we have that $\left.f\right|_{\mathcal{V}}$ in the above is isometrically rigid if and only if each factor is rigid.

Corollary 2. Let $f: M^{n} \rightarrow \mathbb{Q}_{c}^{n+p}, 2 p \leq n$, be an isometric immersion of a connected Riemannian manifold $M^{n}$ with $K_{M} \leq c$ and Ricci curvature Ric R $_{M}<c$. Then $c=0, n=2 p$ and $f$ splits locally as a product of $p$ negatively curved surfaces of $\mathbb{R}^{3}$. Moreover, the splitting is global provided that $M^{n}$ is a Hadamard manifold.

The assumption on the Ricci curvature in the above can be replaced by the weaker one $\nu=0$. Also, the Hadamard condition can probably be relaxed a bit. Combining our results and $[\mathrm{Z}]$, we can state the complex analogue of the above:

Theorem 3. Let $X^{n}$ be an immersed complex submanifold of $\mathbb{C} \mathbb{Q}_{c}^{n+p}$, the complex space form of constant holomorphic sectional curvature c. Assume that $X^{n}$ has nonpositive extrinsic sectional curvature. Then the index of relative nullity of $X^{n}$ satisfies $\nu \geq n-p$ and:

(1) when $\nu=n-p=0$, we must have $c=0$;

(2) when $c=0$ and $\nu=n-p, X^{n}$ is locally holomorphically isometric to a product

$$
\mathbb{C}^{k} \times X^{n_{1}} \times \cdots \times X^{n_{p}} \subseteq \mathbf{X}^{n+p}, \quad n=k+\sum_{i=1}^{p} n_{i},
$$

for some $0 \leq k \leq \nu$, where each $X^{n_{i}} \subseteq \mathbb{C}^{n_{i}+1}$ is a nowhere flat nonpositively curved hypersurface. 
Moreover, if $X^{n}$ is complete, then its universal covering is holomorphically isometric to the product $\mathbb{C}^{\nu} \times \Sigma_{1} \times \cdots \times \Sigma_{p}$, where each $\Sigma_{i} \hookrightarrow \mathbb{C}^{2}$ is a complete immersion of the unit disc. All dimensions here are the complex ones.

Notice that the real analyticity of $X^{n}$ prevented $k$ from jumping around. The last part of Theorem 3 is because, by a theorem of Abe in $[\mathrm{A}]$, any complete immersed complex submanifold of $\mathbb{C}^{m}$ with one dimensional Gauss image must be a cylinder.

Remark. Any Euclidean hypersurface $g: H^{m} \rightarrow \mathbb{R}^{m+1}$ of nonpositive sectional curvature without flat points can be described locally by means of the Gauss parametrization in the following way (see $[D G]$ for details). Take a surface $\xi$ : $V^{2} \rightarrow \mathbb{S}^{m}$ in the Euclidean unit sphere and a smooth function $\gamma$ on $V^{2}$. The map $\Psi: T_{\xi}^{\perp} V \rightarrow \mathbb{R}^{m+1}$ given by

$$
\Psi(v)=\gamma \xi+\operatorname{grad} \gamma+v
$$

parametrizes $g$ over the normal bundle of $\xi$, in the open set of normal vectors $v$ which satisfies $\operatorname{det}\left(\gamma \operatorname{Id}+\operatorname{Hess}_{\gamma}-B_{v}\right)<0$. Here, $B_{v}$ denotes the second fundamental operator of $\xi$ in the direction $v$. In this parametrization, $\xi$ is the Gauss map of $g$ and $\gamma=\langle g, \xi\rangle$ its support function. For a discussion on the isometric deformations of those hypersurfaces see [DFT]. Observe that any isometric immersion $f$ as in Theorem 1 can now be explicitly parametrized locally along $\mathcal{U}$ using the Gauss parametrization for each factor.

\section{The flatness of the normal bundle}

Let $\alpha: V^{n} \times V^{n} \rightarrow W^{p}$ be a symmetric bilinear map, where $V$ and $W$ are real vector spaces of dimension $n$ and $p$, respectively, and $W$ is equipped with an inner product $\langle$,$\rangle . Assume \alpha$ is nonpositive as defined in [F1], i.e.,

$$
K_{\alpha}(X, Y)=\langle\alpha(X, X), \alpha(Y, Y)\rangle-\|\alpha(X, Y)\|^{2} \leq 0,
$$

for all $X, Y \in V$. Denote by $\nu$ the dimension of the null space $N$ of $\alpha$ :

$$
N=\{X \in V \mid \alpha(X, Y)=0, \forall Y \in V\} .
$$

Recall that a subspace $T \subseteq V$ is said to be asymptotic, if $\alpha(X, Y)=0$ for all $X, Y \in T$. We know from [F1] that, for the above $\alpha, \nu \geq n-2 p$. The main technical part of this article is the following diagonalization result for the borderline case $\nu=n-2 p$.

Proposition 4. Let $\alpha: V^{n} \times V^{n} \rightarrow W^{p}$ be a symmetric, nonpositive bilinear map. If $\nu=n-2 p$, then there exist a basis $\left\{e_{1}, \ldots, e_{n}\right\}$ of $V$ and an orthonormal 
basis $\left\{w_{1}, \ldots, w_{p}\right\}$ of $W$ such that $\left\{e_{2 p+1}, \ldots, e_{n}\right\}$ is a basis of the null space $N$, and for each $i, j \leq 2 p$,

$$
\alpha\left(e_{i}, e_{j}\right)=\delta_{i j}(-1)^{i} w_{\left[\frac{i+1}{2}\right]}
$$

Proof.We will carry out the induction on $p$. When $p=1, \alpha$ is just a symmetric bilinear form, so it can always be diagonalized. The nonpositivity condition will force the rank of $\alpha$ to be less or equal than 2, and when it equals 2, the two nonzero eigenvalues must be of opposite sign. Now assume that the result holds when dim $W<p$, and consider the case $\operatorname{dim} W=p$.

By restricting $\alpha$ to a subspace $\widetilde{V}^{2 p}$ such that $V=N \oplus \widetilde{V}$, we may assume that $n=2 p$ and $\nu=0$. Denote by $\alpha_{X}$ the endomorphism $\alpha_{X}(Y)=\alpha(X, Y)$. By Proposition 6 of [F1] we know that there exists an asymptotic subspace $T^{p} \subseteq \widetilde{V}^{2 p}$ of $\alpha$. Set

$$
r=\min \left\{\operatorname{rank} \alpha_{X}: 0 \neq X \in T\right\}>0 .
$$

Fix a vector $X \in T$ with rank $\alpha_{X}=r$ and let $V^{\prime}=\operatorname{Ker}\left(\alpha_{X}\right) \supseteq T$. Thus, by the first claim in the proof of Proposition 6 of [F1], we know that the image $\alpha\left(V^{\prime} \times V^{\prime}\right)$ is perpendicular to the image subspace $\operatorname{Im}\left(\alpha_{X}\right)$, that is, we have the restriction map

$$
\left.\alpha\right|_{V^{\prime} \times V^{\prime}}: V^{\prime} \times V^{\prime} \rightarrow \operatorname{Im}\left(\alpha_{X}\right)^{\perp} .
$$

Let $N^{\prime}$ be its null space. If there is $Y \in N^{\prime} \backslash T$, then $\operatorname{span}(T \cup\{Y\})$ would be an asymptotic subspace of $\alpha$ of dimension $p+1$. By Proposition 8 of [F1], we get $\nu \geq 1$, a contradiction to our assumption. Therefore, $N^{\prime} \subseteq T$.

For each $Y \in N^{\prime} \subseteq T$, we have $\operatorname{Ker}\left(\alpha_{Y}\right) \supseteq V^{\prime}=\operatorname{Ker}\left(\alpha_{X}\right)$, so rank $\alpha_{Y}=r$. Therefore,

$$
V^{\prime}=\operatorname{Ker}\left(\alpha_{Y}\right), \quad \forall 0 \neq Y \in N^{\prime} .
$$

Put $W_{0}=\operatorname{span}\left\{\operatorname{Im}\left(\alpha_{Y}\right): Y \in N^{\prime}\right\}$ which has dimension $r+s$, for some $s \geq$ 0 . Again from the proof of Proposition 6 of [F1], we know that $\alpha\left(V^{\prime} \times V^{\prime}\right)$ is perpendicular to $W_{0}$, that is,

$$
\beta=\left.\alpha\right|_{V^{\prime} \times V^{\prime}}: V^{\prime} \times V^{\prime} \rightarrow W_{0}^{\perp}
$$

is itself a symmetric, nonpositive bilinear map, with $\operatorname{dim} V^{\prime}=2 p-r, \operatorname{dim} W_{0}^{\perp}=$ $p-r-s$. Write $q=\operatorname{dim} N^{\prime}$. Then by Proposition 9 of [F1] we have

$$
q \geq(2 p-r)-2(p-r-s)=r+2 s .
$$

On the other hand, if $\left\{Y_{1}, \ldots, Y_{q}\right\}$ is a basis of $N^{\prime}$ and $Z \in V \backslash V^{\prime}$, from (1) we obtain that the set of vectors $\left\{\alpha\left(Y_{1}, Z\right), \cdots, \alpha\left(Y_{q}, Z\right)\right\}$ in $W_{0}$ must be linearly independent. Thus

$$
q \leq r+s
$$

We conclude from (2) and (3) that $s=0$ and $q=r$. So we can apply the induction hypothesis on $\beta$. However, we want to show first that $r=1$. 
Assume the contrary, that is, $q>1$. Take a subspace $V_{1}^{r}$ such that $V_{1} \oplus V^{\prime}=V$. Choose any $Y \in N^{\prime}$ not collinear with $X$. Since $s=0$, (the restriction of) both $\alpha_{X}$ and $\alpha_{Y}$ give isomorphisms between $V_{1}$ and $W_{0}^{\perp}$. Fix an orthonormal basis $\left\{w_{1}, \ldots, w_{r}\right\}$ of $W_{0}^{\perp}$. Let $\left\{v_{1}, \ldots, v_{r}\right\}$ be the basis of $V_{1}$ such that $\alpha_{X}\left(v_{i}\right)=w_{i}$ and write $\alpha_{Y}\left(v_{i}\right)=\sum_{j=1}^{r} B_{i j} w_{j}$. That is, we identify $V_{1}$ and $W_{0}^{\perp}$ through $\alpha_{X}$, and use the matrix $B$ to represent $\alpha_{Y}$.

If $B$ has a real eigenvalue $\lambda$, then $\alpha_{Y-\lambda X}$ would have rank less than $r$, which contradicts (1). So the matrix $B$ has no real eigenvalues. By considering a complex eigenvector which corresponds to a complex eigenvalue of $B$, we obtain two 2planes $P \subseteq V_{1}, Q \subseteq W_{0}^{\perp}$, such that both $\alpha_{X}$ and $\alpha_{Y}$ give isomorphisms between $P$ and $Q$.

Now let us fix an orthonormal basis $\left\{w_{1}, w_{2}\right\}$ of $Q$, and let $\left\{e_{3}, e_{4}\right\}$ be the basis of $P$ such that $\alpha_{X}\left(e_{3}\right)=w_{1}, \alpha_{X}\left(e_{4}\right)=w_{2}$. Write

$$
\alpha_{Y}\left(e_{3}\right)=a w_{1}+b w_{2}, \quad \alpha_{Y}\left(e_{4}\right)=c w_{1}+d w_{2} .
$$

Replacing $Y$ by $Y-d X$, we may assume that

$$
d=0 .
$$

We know that the $2 \times 2$ real matrix with entries $a, b, c, 0$ can not have any real eigenvalue, or equivalently,

$$
4 b c+a^{2}<0
$$

Set $e_{1}=X, e_{2}=Y$. For arbitrary real constants $x$ and $y$, let us consider the vectors $Z=x e_{1}+x y e_{2}+x e_{3}-e_{4}$ and $Z^{\prime}=y e_{2}+e_{3}$. We have

$$
Z \wedge Z^{\prime}=x y e_{1} \wedge e_{2}+x e_{1} \wedge e_{3}+y e_{2} \wedge e_{4}+e_{3} \wedge e_{4} .
$$

Define the symmetric bilinear form $R$ on $\Lambda^{2} V$, the curvature of $\alpha$, as

$$
R\left(Z_{1} \wedge Z_{2}, Z_{3} \wedge Z_{4}\right)=\left\langle\alpha\left(Z_{1}, Z_{3}\right), \alpha\left(Z_{2}, Z_{4}\right)\right\rangle-\left\langle\alpha\left(Z_{1}, Z_{4}\right), \alpha\left(Z_{2}, Z_{3}\right)\right\rangle .
$$

Hence, the matrix of $R$ under the partial basis $\left\{e_{1} \wedge e_{2}, e_{1} \wedge e_{3}, e_{2} \wedge e_{4}, e_{3} \wedge e_{4}\right\}$ is

$$
R=\left[\begin{array}{cccc}
0 & 0 & 0 & c-b \\
0 & -1 & -b & -f \\
0 & -b & -c^{2} & -g \\
c-b & -f & -g & -h
\end{array}\right]
$$

Therefore $-R\left(Z \wedge Z^{\prime}, Z \wedge Z^{\prime}\right)=x^{2}+c^{2} y^{2}+h+2(2 b-c) x y+2 f x+2 g y$. Thus, the nonpositivity of $\alpha$ gives us

$$
c^{2} y^{2}+2((2 b-c) x+g) y+\left(x^{2}+2 f x+h\right) \geq 0 .
$$


Hence, the discriminant with respect to $y$ must be nonpositive, that is,

$\left.0 \leq c^{2}\left(x^{2}+2 f x+h\right)-((2 b-c) x+g)\right)^{2}=\left(4 b c-4 b^{2}\right) x^{2}+2\left(c^{2} f+c g-2 b g\right) x+\left(c^{2} h-g^{2}\right)$.

Since $a^{2}+4 b c<0$, the leading coefficient is negative, which is a contradiction for $x$ sufficiently large. This completes the proof of the claim that $q=r=1$.

Now applying the induction hypothesis on the restriction map $\beta$, we obtain an orthonormal basis $\left\{w_{1}, \ldots, w_{p}\right\}$ of $W$ and a basis $\left\{e_{1}^{\prime}, e_{2}, e_{2}^{\prime}, \ldots, e_{p}, e_{p}^{\prime}\right\}$ of $V^{\prime}=\operatorname{Ker}\left(\alpha_{X}\right)$ such that $X=e_{1}^{\prime}, \operatorname{Im}\left(\alpha_{X}\right)=\operatorname{span}\left\{w_{1}\right\}$,

$$
\alpha\left(e_{i}, e_{j}\right)=\delta_{i j} w_{i}, \quad \alpha\left(e_{i}^{\prime}, e_{j}^{\prime}\right)=-\delta_{i j} w_{i}, \quad \alpha\left(e_{i}, e_{j}^{\prime}\right)=0, \quad \forall 2 \leq i, j \leq p,
$$

and of course $\alpha\left(e_{1}^{\prime}, e_{1}^{\prime}\right)=\alpha\left(e_{1}^{\prime}, e_{i}\right)=\alpha\left(e_{1}^{\prime}, e_{i}^{\prime}\right)=0$, for all $2 \leq i \leq p$.

Choose a vector $e_{1} \in V \backslash V^{\prime}$ such that $\alpha\left(e_{1}, e_{1}^{\prime}\right)=w_{1}$. Write $\alpha=\left(A^{1}, \ldots, A^{p}\right)$, where each $A_{a b}^{k}=\left\langle\alpha\left(e_{a}, e_{b}\right), w_{k}\right\rangle$ is a symmetric $2 p \times 2 p$ matrix. Here for convenience we adopt the notations $e_{i}^{\prime}=e_{p+i}$ and $i^{\prime}=i+p$, for $i \leq p$. Under the basis $\left\{e_{a} \wedge e_{b} ; 1 \leq a<b \leq 2 p\right\}$ of $\Lambda^{2} V$, the coordinate matrix of the bilinear form $R$ becomes

$$
R_{a b, c d}=\sum_{k=1}^{p}\left(A_{a c}^{k} A_{b d}^{k}-A_{a d}^{k} A_{b c}^{k}\right) .
$$

The nonpositivity of $\alpha$ simply says that $R\left(Z_{1} \wedge Z_{2}, Z_{1} \wedge Z_{2}\right) \leq 0$. For any three vectors $Z_{i}, i=1,2,3$, by considering the nonpositivity at $Z_{1} \wedge\left(Z_{2}+x Z_{3}\right)$ for arbitrary $x$, we have

$$
R\left(Z_{1} \wedge Z_{2}, Z_{1} \wedge Z_{2}\right) \cdot R\left(Z_{1} \wedge Z_{3}, Z_{1} \wedge Z_{3}\right) \geq\left(R\left(Z_{1} \wedge Z_{2}, Z_{1} \wedge Z_{3}\right)\right)^{2} .
$$

For all $2 \leq i \leq p$ and $2 \leq a \neq i, i^{\prime}$, from the above and $R_{i a, i a}=0$ we have $R_{1 i, i a}=-A_{1 a}^{i}=0$. That is, $A_{1 j}^{i}=A_{1 j^{\prime}}^{i}=0$, for all $2 \leq i \neq j \leq p$. Replacing $e_{1}$ by $e_{1}-\sum_{i=2}^{p}\left(A_{1 i}^{i} e_{i}-A_{1 i^{\prime}}^{i} e_{i}^{\prime}\right)$, we may assume that

$$
A_{1 j}^{i} \equiv 0, \quad \forall i, j \geq 2 .
$$

For $2 \leq i \leq p$, set

$$
b_{i}=A_{11}^{i}, a_{i}=A_{1 i}^{1}, c_{i}=A_{1 i^{\prime}}^{1}
$$

Thus,

$$
\begin{gathered}
R_{11^{\prime}, 11^{\prime}}=-1, \\
R_{1 i, 1 i}=b_{i}-a_{i}^{2}, \quad R_{11^{\prime}, 1 i}=-a_{i}, \\
R_{1 i^{\prime}, 1 i^{\prime}}=b_{i}-c_{i}^{2}, \quad R_{11^{\prime}, 1 i^{\prime}}=-c_{i},
\end{gathered}
$$

since $A_{11^{\prime}}^{1}=1$. From (5) and $R_{11^{\prime}, 11^{\prime}} R_{1 i, 1 i} \geq\left(R_{11^{\prime}, 1 i}\right)^{2}$ we get $b_{i} \leq 0$. Similarly, replacing $i$ by $i^{\prime}$, we have $b_{i} \geq 0$. Therefore, all $b_{i}=0$. 
Now we take any nonsingular $2 \times 2$ matrix

$$
\left[\begin{array}{ll}
a & b \\
c & d
\end{array}\right]
$$

such that

$$
\left[\begin{array}{ll}
a & c \\
b & d
\end{array}\right]\left[\begin{array}{cc}
A_{11}^{1} & 1 \\
1 & 0
\end{array}\right]\left[\begin{array}{ll}
a & b \\
c & d
\end{array}\right]=\left[\begin{array}{cc}
1 & 0 \\
0 & -1
\end{array}\right]
$$

and set

$$
\tilde{e}_{1}=a e_{1}+c e_{1}^{\prime}, \quad \tilde{e}_{1}^{\prime}=b e_{1}+d e_{1}^{\prime}, \quad \tilde{e}_{i}=e_{i}-a_{i} e_{1}^{\prime}, \quad \tilde{e}_{i}^{\prime}=e_{i}^{\prime}-c_{i} e_{1}^{\prime}, \quad 2 \leq i \leq p .
$$

Then under the new basis $\left\{\tilde{e}_{a}\right\}$ of $V$, we have $\alpha\left(\tilde{e}_{a}, \tilde{e}_{b}\right)=0$, if $a \neq b, b^{\prime}$, and

$$
\alpha\left(\tilde{e}_{i}, \tilde{e}_{i}\right)=w_{i}, \quad \alpha\left(\tilde{e}_{i}^{\prime}, \tilde{e}_{i}^{\prime}\right)=-w_{i}, \quad \forall 1 \leq i \leq p .
$$

This completes the proof of Proposition 4.

Let us examine the diagonalizing frame $\left\{w_{i}\right\}$ of Proposition 4. Set

$$
\mathcal{D}=\left\{X \in V: \operatorname{rank}\left(\alpha_{X}\right) \leq 1\right\} .
$$

This set of course depends only on $\alpha$. By Proposition 4 , we know that $\mathcal{D}$ is the union of $p$ subspaces of dimension $\nu+2$, denoted by $\mathcal{D}_{i}, i=1, \ldots, p$, with $\mathcal{D}_{i} \cap \mathcal{D}_{j}=N$ for all $i \neq j$. If we choose a plane $V_{i} \subseteq \mathcal{D}_{i}$ which has trivial intersection with $N$, then $V$ is the direct sum

$$
V=N \oplus V_{1} \oplus \cdots \oplus V_{p}
$$

and $\alpha\left(\mathcal{D}_{i} \times \mathcal{D}_{j}\right)=0$ if $i \neq j$, while all $\alpha\left(\mathcal{D}_{i} \times \mathcal{D}_{i}\right)$ are one dimensional and mutually perpendicular. So the orthonormal frame $\left\{w_{i}\right\}$ is uniquely determined up to permutations.

It is interesting to note that $K \leq 0$ does not implies in general that the symmetric curvature operator $R$ is negative semidefinite. However, it is easy to see using Proposition 4 that, in our case, we really have $R \leq 0$. In fact, $\left\{e_{i} \wedge e_{i+p}: 1 \leq i \leq p\right\}$ is a basis of the orthogonal complement $F$ of the nullity space of $R$ in $\Lambda^{2} V$ formed by the unique (up to scaling) decomposable elements in $F$. Indeed, $e_{i} \wedge e_{i+p}$ is eigenvector of $R$ of eigenvalue $K\left(e_{i}, e_{i+p}\right) \neq 0$.

We are now in position to give the remaining proofs.

Proofs of Theorem 1 and Corollary 2. For each $x \in M^{n}$, consider $\alpha_{f}(x)$ the vector valued second fundamental form of $f$ at $x$. Since $K_{M} \leq 0$, the Gauss equation tells us that $\alpha_{f}(x)$ is nonpositive. Thus, we apply Proposition 4 to it to obtain the 
special (smooth) orthonormal frame $\left\{w_{i}, 1 \leq i \leq p\right\}$. By Theorem 1 and Corollary 2 of [F2], we only need to prove that the normal bundle of $f$ is flat. We will show indeed that this frame is normal parallel.

For each $1 \leq i \leq p$, consider the shape tensor $A_{w_{i}}$ on $M^{n}$ defined by $\left\langle A_{w_{i}} X, Y\right\rangle=$ $\left\langle\alpha_{f}(X, Y), w_{i}\right\rangle$. By Proposition $4, V_{i}=\operatorname{Im} A_{w_{i}}$ are two dimensional distributions on $M^{n}$ such that

$$
V_{1} \oplus \cdots \oplus V_{p}=\Delta^{\perp}
$$

where $\Delta$ stands for the relative nullity distribution of $f$. Let $\psi_{i j}$ be the 1 -forms defined by $\psi_{i j}(X)=\left\langle\nabla_{X}^{\perp} w_{i}, w_{j}\right\rangle$. We only need to show that $\psi_{i j}=0$, for all $i, j$.

Recall that the Codazzi equation for $A_{w_{i}}$ is

$$
\nabla_{X}\left(A_{w_{i}} Y\right)-A_{w_{i}} \nabla_{X} Y-A_{\nabla_{X}^{\frac{1}{X} w_{i}}} Y=\nabla_{Y}\left(A_{w_{i}} X\right)-A_{w_{i}} \nabla_{Y} X-A_{\nabla_{\frac{1}{Y} w_{i}}} X .
$$

Taking in (8) $X, Y \in V_{i}^{\perp}=\operatorname{Ker} A_{w_{i}}$ we easily obtain using (7) that

$$
A_{w_{j}}\left(\psi_{i j}(X) Y-\psi_{i j}(Y) X\right)=0, \quad \forall X, Y \in V_{i}^{\perp}, \quad 1 \leq j \leq p .
$$

Suppose that there is $X_{0} \in V_{i}^{\perp}$, and $j \neq i$ such that $\psi_{i j}\left(X_{0}\right) \neq 0$. The above equation implies that $V_{i}^{\perp} \subset V_{j}^{\perp} \oplus$ span $\left\{X_{0}\right\}$, that is,

$$
T_{x} M \neq V_{i}^{\perp}+V_{j}^{\perp}=\left(V_{i} \cap V_{j}\right)^{\perp},
$$

which is a contradiction by (7). Thus $V_{i}^{\perp} \subset \operatorname{Ker} \psi_{i j}$, for all $i, j$. By the orthonormality of $\left\{w_{i}\right\}$ we have $\psi_{i j}=-\psi_{j i}$. Therefore, $T_{x} M=V_{i}^{\perp}+V_{j}^{\perp} \subset \operatorname{Ker} \psi_{i j}$. Notice that the Ricci equations imply that the $V_{i}$ 's are orthogonal. This concludes our proof.

The proof of Theorem 3 can be obtained by combining the diagonalization theorem of $[\mathrm{Z}]$ (together with the similar argument of the orthogonality of the special frame) and the proof of the Theorem 1 of [F2]. So we shall omit it here.

\section{Final comments}

i) Let us explain Theorem 1 a little bit. We have everywhere on $M^{n}$ the orthogonal decomposition $T M=N \oplus V_{1} \oplus \cdots \oplus V_{p}$ of the tangent bundle into distributions. Let $\widetilde{V}_{i}$ be the distribution spanned by all vector fields in $V_{i}$ and all $\nabla_{X_{1}} \cdots \nabla_{X_{s}} X_{s+1}$, where all $X_{j} \in V_{i}$. It is shown in [F2] that $\widetilde{V}_{i} \perp \widetilde{V}_{j}$ whenever $i \neq j$, and all $\widetilde{V}_{i}$ are parallel distributions (in the neighborhood where they have constant dimensions). Let $n_{i}(x)$ be the dimension of $\widetilde{V}_{i}$ at $x$. Each $n_{i}$ is a lower semicontinuous integervalued function. If $k=n-\sum_{i=1}^{p} n_{i}$, then $0 \leq k \leq \nu$. Let $\mathcal{U}$ be the open dense subset of $M^{n}$ which is the disjoint union of open subsets $\mathcal{U}_{j}$ in which $k(x)$ takes constant value $j$. All $n_{i}$ are necessarily constant in $\mathcal{U}_{j}$, and we have the desired 
local splitting on $\mathcal{U}_{j}$. Observe that, using the Gauss parametrization, it is easy to construct examples of submanifolds with the functions $n_{i}$ nonconstant. Therefore, for $\nu>0$ we can only obtain the local splitting along an open dense subset. With this is mind, the same argument as in Corollary 2 of [F2] proves the following

Theorem 5. Let $f: M^{n} \rightarrow \mathbb{Q}_{c}^{2 n-r}, 2 \leq r \leq n / 2$, be an isometric immersion with flat normal bundle of a connected Riemannian manifold with $K_{M} \leq c$ and Ric $_{M}<c$. Then $c=0$ and $f$ splits locally as a product of $r$ nonpositively curved Euclidean submanifolds, that is, $f=f_{1} \times \cdots \times f_{r}$ locally, with $f_{i}: M_{i}^{n_{i}} \rightarrow \mathbb{R}^{2 n_{i}-1}$. The splitting is global provided $M^{n}$ is a Hadamard manifold.

Again, the assumption on the Ricci curvature can be replaced by $\nu=0$.

ii) We believe that the case $\nu=n-2 p>0$ for an isometric immersion $f$ : $M^{n} \rightarrow \mathbb{Q}_{c}^{n+p}$, with $c \neq 0$, cannot occur. It would be interesting either to prove its nonexistence or to construct such an example. The complex case should be similar.

iii) Taking the curvature tensor $R$ as a 4 -tensor on $M^{n}$, it is defined the nullity space of $M^{n}$ at $x$ as the subspace $\Gamma(x)=\left\{X \in T_{x} M: R(X, Y, Z, W)=\right.$ $\left.0, \forall Y, Z, W \in T_{x} M\right\}$. This is an intrinsic subspace, so its dimension $\mu(x)$ called the nullity index of $M^{n}$ is an intrinsic function. For an isometric immersion $f$ of $M^{n}$ into Euclidean space we always have that the relative nullity distribution $\Delta$ of $f$ satisfies $\Delta \subset \Gamma$. Thus, our assumption on the relative nullity distribution in Theorem 1 can be replaced by the intrinsic one $\mu=n-2 p$. The same holds for Corollary 2.

iv) Now let us consider the more general situation discussed in Theorem 1 of [F2], namely, $\nu=n-p-r$, for some $2 \leq r \leq p$. It is natural to ask if it can be generalized by dropping the flatness of the normal bundle assumption as we did for the case $r=p$. The answer to this question seems to be negative, since the algebraic decomposition Proposition 4 does not generalizes, even for the case $r=p-1$, as the following example shows. Take $A_{i}$ defined as

$$
A_{1}=\left[\begin{array}{ccccc}
1 & 0 & 0 & 0 & 0 \\
0 & -1 & 0 & 0 & 0 \\
0 & 0 & 0 & 0 & 0 \\
0 & 0 & 0 & 0 & 0 \\
0 & 0 & 0 & 0 & 0
\end{array}\right], A_{2}=\left[\begin{array}{ccccc}
0 & 0 & 0 & 0 & 0 \\
0 & 0 & 0 & 0 & 0 \\
0 & 0 & 1 & 0 & 0 \\
0 & 0 & 0 & -1 & 0 \\
0 & 0 & 0 & 0 & 0
\end{array}\right], A_{3}=\left[\begin{array}{ccccc}
0 & 0 & 0 & 0 & 1 \\
0 & 0 & 0 & 0 & 1 \\
0 & 0 & 0 & 0 & 1 \\
0 & 0 & 0 & 0 & 1 \\
1 & 1 & 1 & 1 & 0
\end{array}\right]
$$

The bilinear form $\alpha=\left(A_{1}, A_{2}, A_{3}\right): \mathbb{R}^{5} \times \mathbb{R}^{5} \rightarrow \mathbb{R}^{3}$ is nonpositive, has $\nu=$ $n-p-r=0$ for $r=p-1=2$ but is not decomposable. It is easy to generalize this example for all $p$. Thus the analogous result to Proposition 4 is false for $\nu=n-p-r$ and $2 \leq r \leq p-1$. 


\section{Acknowledgement}

We would like to thank Professor M. Dajczer for his interests and helpful conversations. We would also like to thank the referee of this article for several valuable suggestions for improvements.

\section{References}

[A] K. Abe, Applications of a Riccati type differential equation to Riemannian manifolds with totally geodesic distributions, Tôhoku Math. J. 25 (1973), 425-444.

[DG] M. Dajczer and D. Gromoll, Gauss parametrizations and rigidity aspects of submanifolds, J. Diff. Geom. 22 (1985), 11-12.

[DFT] M. Dajczer, L. Florit and R. Tojeiro, On deformable hypersurfaces in space forms, preprint.

[F1] L. Florit, On submanifolds with nonpositive extrinsic curvature, Math. Ann. 298 (1994), 187-192.

[F2] L. Florit, A splitting theorem for Euclidean submanifolds of nonpositive sectional curvature, Math. Z. 225(4) (1997), 685-690.

[M] J. Moore, On isometric immersions of Riemannian products, J. Diff. Geom. 5 (1971), $159-168$

[Z] F. Zheng, Isometric embedding of Kähler manifolds with nonpositive sectional curvature, Math. Ann. 304 (1996), 769-784.

Luis A. Florit

IMPA

Estrada Dona Castorina, 110

22460-320 Rio de Janeiro - Brazil

e-mail: luis@impa.br

(Received: October 21, 1997)
Fangyang Zheng

The Ohio State University

Columbus, OH 43210-1174 - USA

e-mail: zheng@math.ohio-state.edu 